\title{
Diacron reactive oxygen metabolites and biological antioxidant potential tests for patients with age-related macular degeneration
}

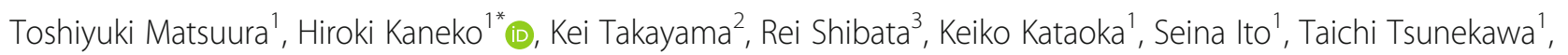
Hideyuki Shimizu', Ayana Suzumura', Rina Namba', Yasuki Ito', Toyoaki Murohara ${ }^{4}$ and Hiroko Terasaki ${ }^{1}$

\begin{abstract}
Background: Previously, we showed that serum malondialdehyde (MDA) was significantly higher in patients with neovascular age-related macular degeneration (nAMD) than in those without AMD. The Diacron reactive oxygen metabolites (d-ROMs) and biological antioxidant potential (BAP) tests are known markers of oxidative stress. The aim of this study was to use d-ROMs and BAP tests to evaluate changes in systemic oxidative stress in patients with nAMD.

Methods: Blood serum samples were collected from 34 patients with nAMD (mean age: $76.5 \pm 7.7$ years; 22 men) and 20 control subjects (mean age: $62.9 \pm 14.0$ years; 10 men), and d-ROMs and BAP tests were examined.

Results: In men, the mean level of $d$-ROMs for the nAMD patients was significantly higher than that for the controls $(312.0 \pm 52.4$ vs. $275.1 \pm 45.5$ U.CARR, respectively; $P<.05)$. There was a significant correlation between d-ROM level and CNV lesion area in the male nAMD group $(r=.42, P=.05)$. There were no significant differences in mean BAP test results between the nAMD patients and controls for either sex (men: $2241 \pm 549 \mathrm{vs} .2136 \pm 246 \mu \mathrm{mol} / \mathrm{L}$; women: $2263 \pm 292$ vs. $2335 \pm 161 \mu \mathrm{mol} / \mathrm{L})$.
\end{abstract}

Conclusion: The d-ROMs test may provide a useful indicator of nAMD in men but not in women.

Keywords: Age-related macular degeneration, Diacron reactive oxygen metabolites, Biological antioxidant potential, Oxidative stress, Choroidal neovascularization

\section{Background}

Age-related macular degeneration (AMD) is a leading cause of blindness, and the increasing number of AMD patients has had a severe economic impact in many developed countries $[1,2]$. Advanced AMD is classified according to its pathophysiology as either neovascular AMD (nAMD) or geographic atrophy (GA) [3]. nAMD is characterized by choroidal neovascularization (CNV) and an increase in intraretinal/subretinal fluid, which is strongly associated with the overexpression of vascular endothelial growth factor (VEGF). Multiple risk factors have been shown to contribute to the pathogenesis of

\footnotetext{
* Correspondence: h-kaneko@med.nagoya-u.ac.jp

${ }^{1}$ Department of Ophthalmology, Nagoya University Graduate School of

Medicine, 65 Tsurumai-cho, Showa-ku, Nagoya 466-8550, Japan

Full list of author information is available at the end of the article
}

nAMD, including obesity [4], hypertension [5], smoking [6], and light exposure [7, 8]; it has been presumed that these act through inducing oxidative stress [9-11]. Previously, we showed that serum malondialdehyde (MDA), a marker of oxidative stress, was significantly higher in patients with nAMD than in those without AMD, and that there was a significant correlation between serum MDA levels and the area of CNV [12].

The Diacron reactive oxygen metabolites (d-ROMs) test for measuring reactive oxygen metabolites (ROMs) in blood has been used for various biological and medical purposes [13, 14]. This test uses the Free Radical Analytical System (Diacron International, Grosseto, Italy) [15-17]. The main component of ROMs is hydroperoxide [16, 17], which causes cell death and tissue damage $[18,19]$. Despite its power as an oxidant,

(c) The Author(s). 2020 Open Access This article is distributed under the terms of the Creative Commons Attribution 4.0 International License (http://creativecommons.org/licenses/by/4.0/), which permits unrestricted use, distribution, and 
Table 1 Characteristics of the subjects in the nAMD and control groups

\begin{tabular}{|c|c|c|c|c|}
\hline & \multicolumn{2}{|l|}{ nAMD } & \multicolumn{2}{|l|}{ Control } \\
\hline & Male & Female & Male & Female \\
\hline Number & 22 & 12 & 10 & 10 \\
\hline Age (years) & $73.9 \pm 7.65$ & $78.5 \pm 7.05$ & $66.7 \pm 10.5$ & $67.3 \pm 15.9$ \\
\hline $\log M A R$ BCVA & $0.44 \pm 0.34$ & $0.55 \pm 0.45$ & $0.23 \pm 0.41$ & $0.21 \pm 0.26$ \\
\hline d-ROM (U.CARR) & $312 \pm 52.4$ & $329 \pm 85.7$ & $275 \pm 45.5$ & $343 \pm 37.1$ \\
\hline BAP $(\mu \mathrm{mol} / \mathrm{L})$ & $2286 \pm 500$ & $2263 \pm 292$ & $2136 \pm 246$ & $2335 \pm 161$ \\
\hline Current smoker (\%) & 22.7 & 8.3 & 20.0 & 10.0 \\
\hline
\end{tabular}

Data are mean \pm SEM

IAMD neovascular age-related macular degeneration, logMAR logarithm of the minimum angle of resolution, $B C V A$ best corrected visual acuity, $d-R O M$

Diacron reactive oxygen metabolite, $B A P$ biological antioxidant potential

hydroperoxide in blood is relatively stable compared to its parent free radicals, and its level can be adequately detected. Use of this analyzer also allows the determination of biological antioxidant potential (BAP) in blood. The BAP test measures the capacity of blood to convert an $\mathrm{Fe}^{3+}$ reagent to $\mathrm{Fe}^{2+}[20,21]$. In combination, the d-ROMs and BAP tests provide a comprehensive evaluation of oxidative stress.

In this study, we hypothesized that the d-ROMs and BAP tests could be useful markers of nAMD. We tested this hypothesis separately in groups of male and female nAMD patients and controls, comparing d-ROM and BAP test results. The purpose of this study was to use the d-ROMs and BAP tests to evaluate changes in systemic oxidative stress, and to investigate the correlation between these markers and clinical condition in patients with nAMD.

\section{Methods}

AMD patients and control subjects

The study included 34 patients with nAMD (22 men and 12 women, mean age $75.5 \pm 7.7$ years), and 20 patients in the control group (10 men and 10 women, mean age $67.0 \pm 13.1$ years). All of the subjects were aged $>50$ in nAMD group and $>40$ years in control group and their axial length was in the range $23.0-26.0 \mathrm{~mm}$. Patients with polypoidal choroidal vasculopathy, retinal angiomatous proliferation, or myopic $\mathrm{CNV}$ were not included in the patient group; only typical nAMD was examined. To avoid factors that may affect the results of the d-ROMs and BAP tests, patients with diabetes, dyslipidemia, hypertension, cerebrovascular disorders, cardiovascular disorders, and cancer, as well as those under extreme stress, were also excluded. The diagnosis of nAMD was established on the basis of age, clinical examination, fundus photography, optical coherence tomography, and fluorescein angiography (FA), as previously described [22, 23]. The control group comprised patients with other ocular diseases, including cataract, retinal detachment, and macular hole, and epiretinal membrane. We conducted this crosssectional study in accordance with the guidelines of the Declaration of Helsinki; the protocol was registered to the Clinical Trial Registry of UMIN (Registered number UMIN000032946, Registered date: Jun 11th, 2018) and approved by the Nagoya University Hospital Ethics Review Board (Number 2012-0340-4). We obtained written informed consent from all participating patients.

\section{Best-corrected visual acuity}

The subjects' best-corrected visual acuity (BCVA) was measured using a standard Japanese visual acuity chart. For the statistical analysis, decimal BCVA was converted to the logarithm of the minimum angle of resolution $(\log M A R)$.

\section{FA imaging and evaluation of CNV area}

FA was recorded for all patients in the nAMD group using cSLO (Heidelberg Retina Angiograph, HRA2; Heidelberg
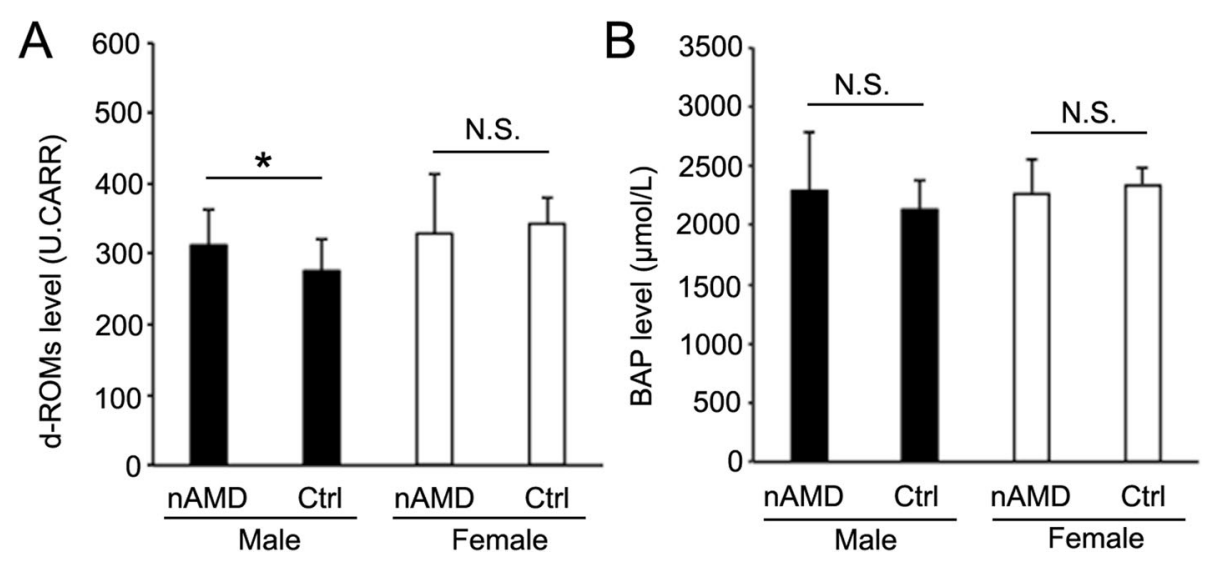

Fig. 1 The mean levels of serum d-ROMs (a) and BAP (b) in male and female patients with neovascular age-related macular degeneration (nAMD) and control subjects (Ctrl). The bars indicate the mean + the standard error of the mean. d-ROMs levels were significantly higher in the male patients with $\mathrm{nAMD}$ than in the male control subjects. ${ }^{*} P<.05$; N.S. = no significant difference. $\mathrm{d}$-ROM=Diacron reactive oxygen metabolite; BAP = biological antioxidant potential 
Engineering, Dossenheim, Germany), as previously described [24-26]. To evaluate the area of the CNV lesion, we traced the border of the area of hyperfluorescein in images captured at $5 \mathrm{~min}$, and counted the pixels using the NAVIS bundled software (Nidek Co. Ltd., Aichi, Japan). The measurements were made by two observers (T.M. and K.T.), both blinded to the subjects' clinical features. The measurements were converted from number of pixels to area (in $\mathrm{mm}^{2}$ ) on the basis that one pixel in the FA image had an area of $.0004 \mathrm{~mm}^{2}$.

\section{The d-ROMs and BAP tests}

A $2 \mathrm{~mL}$ serum sample was collected from each subject and serums were divided into tubes and stored at $80^{\circ} \mathrm{C}$ until use. They were thawed only once before examination.. The indicators of oxidative stress were measured using the free radical analyzer FREE CARRIO DUO (Diacron International). The serum sample was placed in a cuvette filled with a buffer, and a colordeveloping chromogen (N,N-diethyl-p-phenylenediamine) was added and mixed in, resulting in the chromogen substrate being oxidized by the free radicals. The color change in the cuvette was measured optically in a photometer and recorded in units of U.CARR. BAP was also measured using the free radical analyzer FREE CARRIO DUO. A chromogen for BAP (a reagent containing trivalent iron; $50 \mu \mathrm{L}$ ) was added to a cuvette and the color-developing concentration was measured using a photometer (with the results recorded in $\mu \mathrm{mol} / \mathrm{L}$ ).

\section{Statistical analysis}

The data are presented as mean \pm the standard error of the mean (SEM). The Mann-Whitney U test was used to compare data between the nAMD and control groups, with separate analyses for the men and women, and Spearman's correlation was used to evaluate the correlation between d-ROMs and BAP values and age, logMAR BCVA, and CNV area. $P<.05$ was considered statistically significant.

\section{Results}

Subject characteristics, and serum d-ROM and BAP levels The subjects' characteristics are presented in Table 1. In men, the mean level of $\mathrm{d}$-ROMs for the nAMD patients was significantly higher than that for the controls $(312.0 \pm 52.4$ vs. $275.1 \pm 45.5$ U.CARR, respectively;

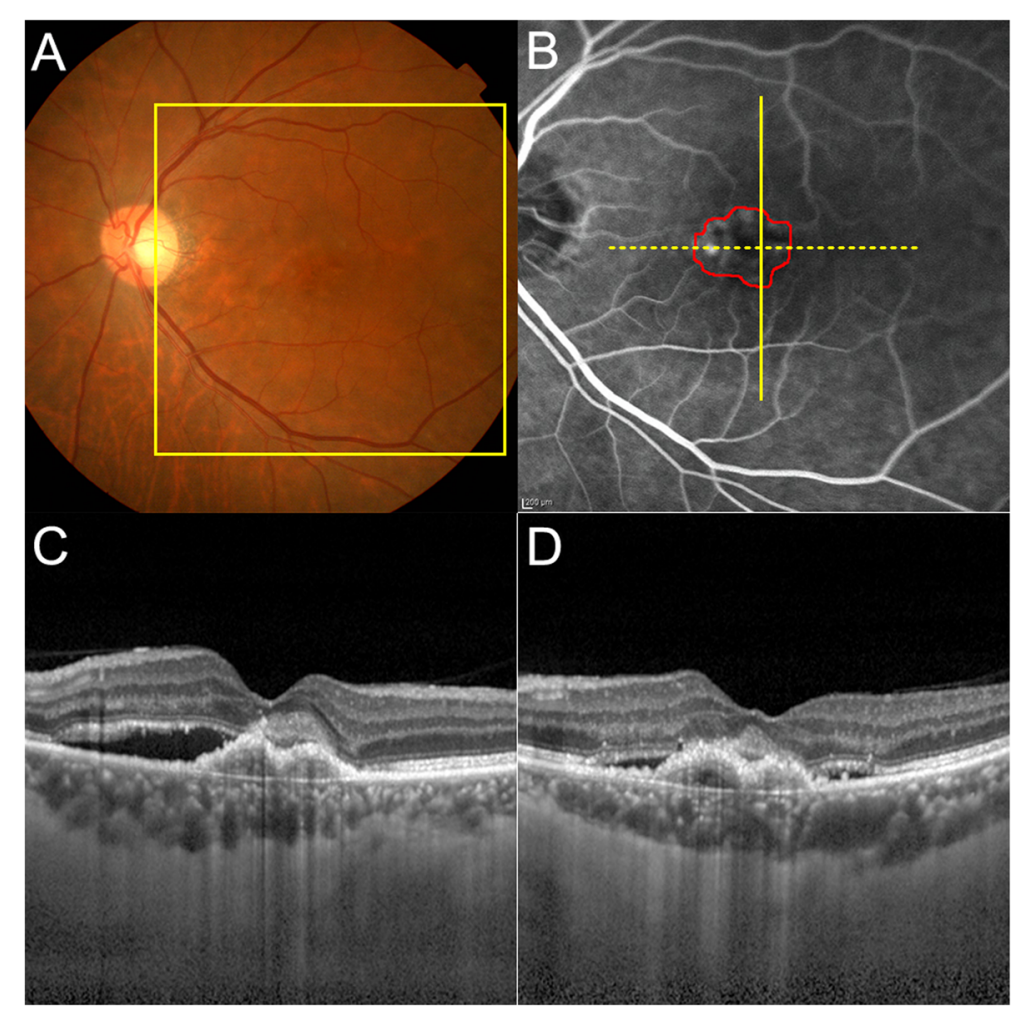

Fig. 2 Representative images from an eye with neovascular age-related macular degeneration (nAMD). a Color fundus image of treatment-naive nAMD. The square box indicates the area shown in (b). b Fluorescein angiography image. The red outline delineates an area of choroidal neovascularization (CNV) that was automatically defined by built-in software. $\mathbf{c}$, d Optical coherence tomography images visualizing the CNV along the solid vertical line and dashed horizontal line in (b), respectively. The following values were measured for this eye: logMAR best corrected visual acuity $=0.22$; baseline serum Diacron reactive oxygen metabolite ( $d$-ROM) level $=339$ U.CARR; serum biological antioxidant potential $(B A P)=2712 \mu \mathrm{mol} / \mathrm{L}$; and CNV area $=1.72 \mathrm{~mm}^{2}$ 
$P<.05$; Fig. 1a). In women, however, there was no significant difference in mean levels of d-ROMs between the nAMD patients and controls $(328.6 \pm 85.7$ vs. $342.5 \pm 37.1$ U.CARR; $P=.16$; Fig. 1a). There were no significant differences in mean BAP test results between the nAMD patient group and control group for either sex (men: $2241 \pm 549$ vs. $2136 \pm 246 \mu \mathrm{mol} / \mathrm{L}, P=.57$; women: $2263 \pm 292$ vs. $2335 \pm 161 \mu \mathrm{mol} / \mathrm{L}, P=.43$ ) (Fig. 1b). There was no significant difference between the male and female nAMD patients in the mean area of CNV lesions $\left(3.05 \pm 2.36 \mathrm{~mm}^{2}\right.$ and $3.15 \pm 3.23 \mathrm{~mm}^{2}$, respectively; $\left.P=.80\right)$.

\section{Correlations between serum d-ROMs levels and nAMD patient characteristics}

We evaluated correlations between d-ROM levels and age, logMAR BCVA, and CNV area separately for the male and female nAMD patients. A representative image of $\mathrm{CNV}$ in eyes with nAMD is shown in Fig. 2. There was a significant correlation between d-ROMs levels and $\mathrm{CNV}$ area in the male nAMD patients $(r=.42, P=.05$; Fig. 3c), but not in the female nAMD patients $(P=.70$; Fig. 3f). In addition, we did not find any significant correlations between $\mathrm{d}$-ROMs levels and age $(P=.35, .44$; Fig. 3a and d) or logMAR BCVA $(P=.53$, .59; Fig. 3b and e) in either sex.

\section{Correlations between serum BAP levels and nAMD patient characteristics}

We evaluated the correlations between BAP levels and age, $\log \mathrm{MAR} B C V A$, and $\mathrm{CNV}$ area. We found no significant correlations between BAP levels and age $(P=.53$ and 0.20 for male and female nAMD patients, respectively; Fig. $4 \mathrm{a}$ and $\mathrm{d}), \operatorname{logMAR}$ BCVA $(P=.48$ and .22 ; Fig. $4 \mathrm{~b}$ and $\mathrm{e})$, or CNV area $(P=.96$ and .25 ; Fig. $4 \mathrm{c}$ and $\mathrm{f})$.

\section{Discussion}

Various diseases involve oxidative stress, including bronchial asthma, myocardial infarction, hypertension, diabetes, and hyperlipidemia [27, 28]. Among the ocular diseases, there has been an accumulation of evidence demonstrating a significant relationship between oxidative stress and AMD, both in vitro and in vivo $[10,29,30]$. We previously demonstrated that MDA, an oxidative stress marker, is not only a marker of AMD, but is also a direct contributor to

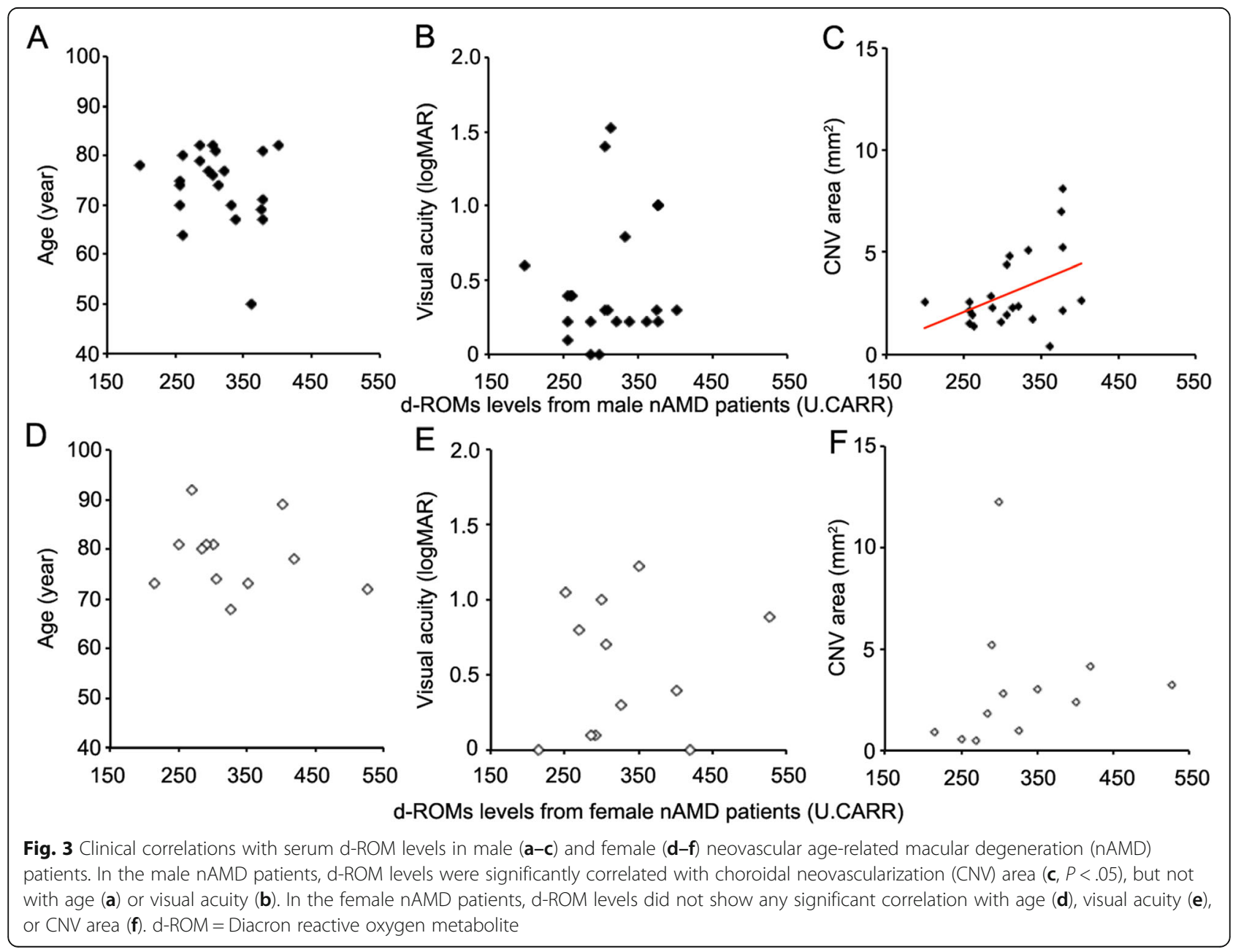




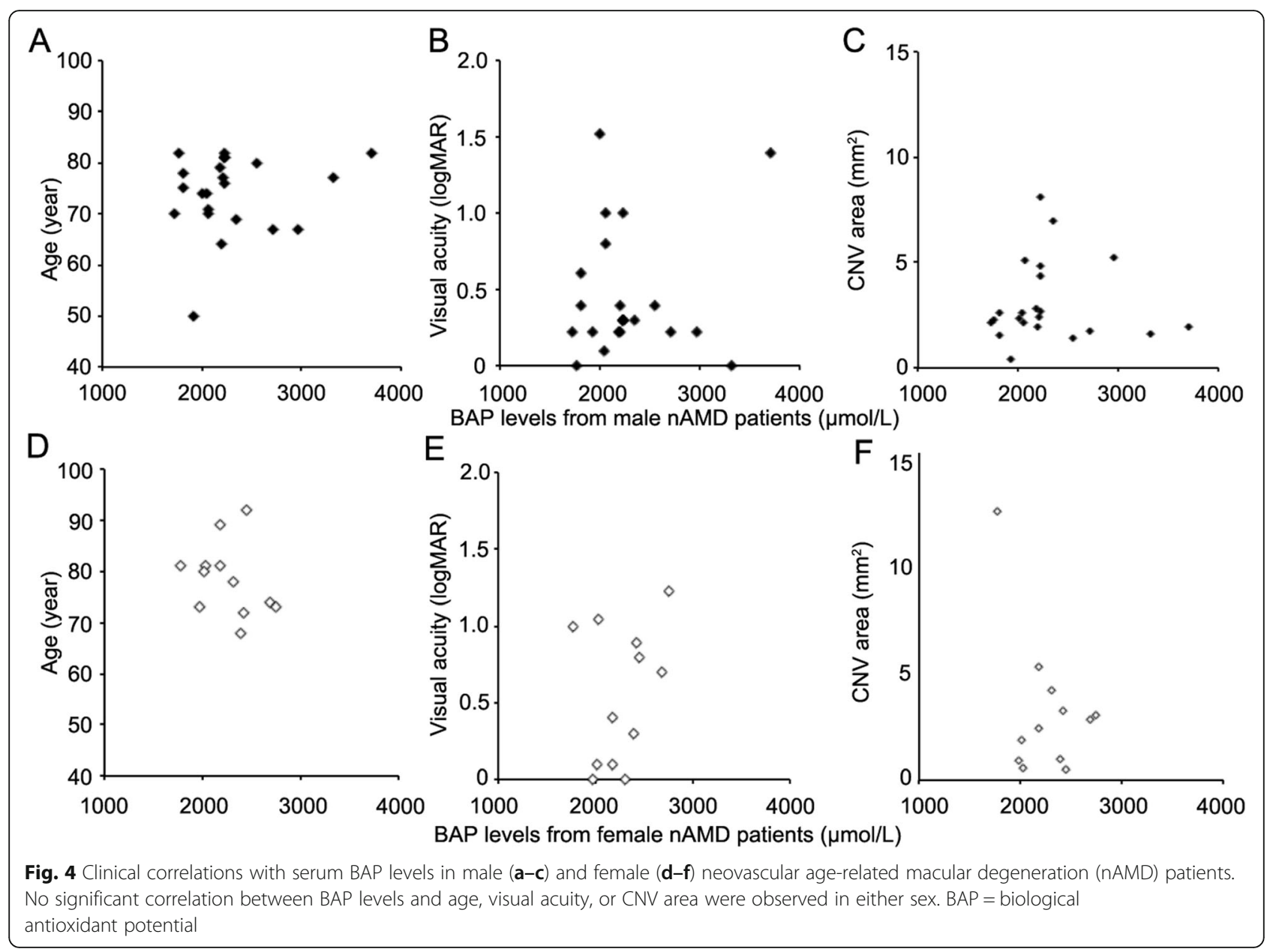

the pathogenesis of AMD [31]. In particular, we showed that serum MDA levels in patients with nAMD were significantly higher than in control subjects, and that there was a significant correlation between serum MDA levels and CNV area [12]. The results of the present study demonstrated that, for the male subjects, serum dROM levels were significantly higher in nAMD patients than in control subjects (Fig. 1), and d-ROM levels were significantly correlated with $\mathrm{CNV}$ area (Fig. 3). These results corroborate our previous findings on MDA and suggest that the d-ROMs test could be a useful indicator of nAMD in male patients.

The study had limitations and some questions remain. (1) We did not find d-ROM levels to have biological importance in female patients with nAMD. Previous studies showed that d-ROM test results were significantly higher in women than in men of the same generation, whereas BAP test results reportedly showed no difference between men and women [32]. d-ROM levels are basically higher in women than in men and, more importantly, they increase with age for both men and women [32]. Because nAMD is a disease of older people, the female nAMD patients necessarily tended to have higher d-ROM levels. (2) We did not find a significant difference in BAP values between the nAMD and control groups for either sex. BAP is defined as the potential for antioxidation. It is possible that the negative result was because the increase in oxidative stress is more important than the decrease in antioxidative potential in the pathogenesis of nAMD. (3) The present study included small numbers. Additionally, there is a difference in age and number among the women and men in the study group. Although d-ROMs were $13.5 \%$ different between nAMD and control in male, those were only $4 \%$ different in female, there is a possibility that difference in results of men and women may be caused by it. Increasing the number of patients may indicate the association of serum d-ROMs with nAMD in women as well. Although there was no statistically significant difference in age in each group, there was variability in the age of each group, and that possibly affected the results of this study. More patients' studies are required to confirm our findings. (4) Serum d-ROM and BAP values are affected by various diseases, diet, anti-oxidant supplements, and smoking etc. In this study, patients with diabetes, dyslipidemia, hypertension, cerebrovascular 
disorders, cardiovascular disorders, and cancer, as well as those under extreme stress, were also excluded, but we did not consider diet and other factors. Therefore, it is possible that oxidative stress increases due to other factors, affecting serum d-ROM and BAP values and further analysis will be required by evaluating confounding factors accurately. There is possibility that the other parameter measured in the regular blood test is associated with the d-ROM levels, and regular blood test is required for the further study for evaluating the importance of $d-R O M$ in AMD. (5) We have reported in the past that MDA is directly involved in increasing CNV size [12]. In this study, it was found that serum d-ROM values and $\mathrm{CNV}$ size correlated significantly in men. However, it is unknown whether d-ROMs increased by oxidative stress is directly involved in the increase in CNV size as with MDA. These issues will be discussed in the future. (6) We previously reported that MDA value could be affected by anti-oxidant supplements. However, in this study, we did not examine the effects of anti-oxidant supplements on d-ROMs and BAP values. Further examination will be beneficial to clarify the relation between anti-oxidant supplements and d-ROMs and BAP values. (7) This study did not examine the correlation between d-ROMs and AMD activity, and there is a possibility that $\mathrm{d}-\mathrm{ROMs}$ values could affect nAMD activity. Investigation of this relationship in the future might be beneficial to control nAMD activity.

The fact that the controls were significantly younger and that the confounders such as smoking (pack-years) and history of antioxidant supplementation was not accounted for in this study and that made the value of our study inherently weak. However, the fact that $\mathrm{d}-\mathrm{ROM}$ s were higher in men with nAMD gives future directions of our study.

\section{Conclusions}

In summary, the results of this study suggest that measuring serum d-ROM levels in male patients could be used as an indicator of CNV severity in nAMD.

\section{Supplementary information}

Supplementary information accompanies this paper at https://doi.org/10. 1186/s12886-020-01334-y.

Additional file 1. Raw data of patient/subject characteristics in this study. $\mathrm{d}-\mathrm{ROM}=$ diacron reactive oxygen metabolite. $\mathrm{BAP}=$ biological antioxidant potential. $C N V=$ choroidal neovascularization. $\log M A R=$ logarithm of the minimum angle of resolution. BCVA = best corrected visual acuity.

\section{Abbreviations}

AMD: Age-related macular degeneration; BAP: Biological antioxidant potential; BCVA: Best-corrected visual acuity; CNV: Choroidal neovascularization; $\mathrm{d}$-ROMs: Diacron reactive oxygen metabolites; FA: Fluorescein angiography; GA: Geographic atrophy; logMAR: Logarithm of the minimum angle of resolution; MDA: Malondialdehyde; ROM: Reactive oxygen metabolites; SEM: Standard error of the mean; VEGF: Vascular endothelial growth factor

\section{Acknowledgments}

The authors thank Drs. Shu Kachi and Tadasu Sugita, for their important clinical suggestions and Mr. Shuji Hirano, Ms. Reona Kimoto, and Ms. Chisato Ishizuka for their technical assistance.

\section{Authors' contributions}

TM, HK, KT, and RS designed and drafted the work, HK, KT, and RS contributed to the conception, KK, TT, HS, AS, RN, YI, TM, and HT interpreted the data, and SI acquired the data. All authors have read and approved the manuscript in its current state.

\section{Funding}

This work was partially supported by Grants-in-Aid for Young Scientist B (K.T; 17 K16964) from the Ministry of Education, Culture, Sports, Science and Technology (http://www.jsps.go.jp/): purchasing assay reagents Japan Foundation for Applied Enzymology (K.T.): purchasing assay reagents.

\section{Availability of data and materials}

All data generated during this study is included in this Additional file 1.

\section{Ethics approval and consent to participate}

We conducted this cross-sectional study in accordance with the guidelines of the Declaration of Helsinki; the protocol was registered to the Clinical Trial Registry of UMIN (Registered number UMIN000032946, Registered date: Jun 11 th, 2018) and approved by the Nagoya University Hospital Ethics Review Board (Number 2012-0340-4). We obtained written informed consent from all participating patients.

\section{Consent for publication}

We obtained written informed consent from all participating patients for using the images for the publication on the condition of anonymity.

\section{Competing interests}

The authors declare that they have no competing interests.

\section{Author details}

${ }^{1}$ Department of Ophthalmology, Nagoya University Graduate School of Medicine, 65 Tsurumai-cho, Showa-ku, Nagoya 466-8550, Japan. ${ }^{2}$ Department of Ophthalmology, National Defense Medical College, Saitama, Japan. ${ }^{3}$ Department of Advanced Cardiovascular Therapeutics, Nagoya University Graduate School of Medicine, Nagoya, Japan. ${ }^{4}$ Department of Cardiology, Nagoya University Graduate School of Medicine, Nagoya, Japan.

Received: 2 April 2019 Accepted: 6 February 2020

Published online: 18 February 2020

References

1. Ambati J, Ambati BK, Yoo SH, lanchulev S, Adamis AP. Age-related macular degeneration: etiology, pathogenesis, and therapeutic strategies. Surv Ophthalmol. 2003;48(3):257-93

2. Bird AC. Therapeutic targets in age-related macular disease. J Clin Invest 2010;120(9):3033-41

3. Dolgin E. Age-related macular degeneration foils drugmakers. Nat Biotechnol. 2017;35(11):1000-1.

4. Zhang QY, Tie LJ, Wu SS, et al. Overweight, obesity, and risk of age-related macular degeneration. Invest Ophthalmol Vis Sci. 2016;57(2):1276-83.

5. Cougnard-Grégoire A, Delyfer MN, Korobelnik JF, et al. Long-term blood pressure and age-related macular degeneration: the ALIENOR study. Invest Ophthalmol Vis Sci. 2013;54(3):1905-12.

6. Joachim N, Mitchell P, Burlutsky G, Kifley A, Wang JJ. The incidence and progression of age-related macular degeneration over 15 years: the Blue Mountains eye study. Ophthalmology. 2015;122(12):2482-9.

7. Takayama K, Kaneko H, Kataoka K, et al. Nuclear factor (erythroid-derived)related factor 2-associated retinal pigment epithelial cell protection under blue light-induced oxidative stress. Oxidative Med Cell Longev. 2016;2016:9.

8. Schick T, Ersoy L, Lechanteur YT, et al. History of sunlight exposure is a risk factor for age-related macular degeneration. Retina. 2016;36(4):787-90. 
9. Bertram KM, Baglole CJ, Phipps RP, Libby RT. Molecular regulation of cigarette smoke induced-oxidative stress in human retinal pigment epithelial cells: implications for age-related macular degeneration. Am J Physiol Cell Physiol. 2009;297(5):C1200-10

10. Du H, Xiao X, Stiles T, Douglas C, Ho D, Shaw PX. Novel mechanistic interplay between products of oxidative stress and components of the complement system in AMD pathogenesis. OJOph. 2016;6(1):43-50.

11. Armstrong RA, Mousavi M. Overview of risk factors for age-related macular degeneration (AMD). J Stem Cells. 2015;10(3):171-91.

12. Matsuura T, Takayama $K$, Kaneko $H$, et al. Nutritional supplementation inhibits the increase in serum malondialdehyde in patients with wet age-related macular degeneration. Oxidative Med Cell Longev. 2017;2017:9548767.

13. Colombini F, Carratelli M, Alberti A. Oxidative stress, d-ROMs test, and ceruloplasmin. Free Radic Res. 2016;50(4):447-53.

14. Ito F, Ito T, Suzuki C, Yahata T, Ikeda K, Hamaoka K. The application of a modified d-roms test for measurement of oxidative stress and oxidized high-density lipoprotein. Int J Mol Sci. 2017;18(2):454.

15. Cesarone MR, Belcaro G, Carratelli M, et al. A simple test to monitor oxidative stress. Int Angiol. 1999;18:127-30.

16. Trotti $R$, Carratelli M, Barbieri $M$, et al. Oxidative stress and a thrombophilic condition in alcoholics without severe liver disease. Haematologica. 2001;86:85-91.

17. Dani C, Martelli E, Bertini G, et al. Plasma bilirubin level and oxidative stress in preterm infants. Arch Dis Child Fetal Neonatal Ed. 2003:88:119-23.

18. Carratelli M, Porcaro L, Ruscica M, De Simone E, Bertelli AAE, Corsi MM. Reactive oxygen metabolites and prooxidant status in children with Down's syndrome. Int J Clin Pharmacol Res. 2001;21:79-84.

19. Yagi $K$, Ohkawa $H$, Ohishi N, Yamashita M, Nakashima T. Lesion of aortic intima caused by intravenous administration of linoleic acid hydroperoxide. J Appl Biochem. 1981;3:58-65.

20. Gerardi G, Usberti M, Martini G, et al. Plasma total antioxidant capacity in hemodialyzed patients and its relationships to other biomarkers of oxidative stress and lipid peroxidation. Clin Chem Lab Med. 2002;40:104-10.

21. Ippolito S, Caruso R, Novembrino C, et al. Dislipidemia and oxidative stress. Ital J Biochem. 2002:51:129.

22. Zajac-Pytrus HM, Pilecka A, Turno-Krecicka A, Adamiec-Mroczek J, Misiuk Hojlo M. The dry form of age-related macular degeneration (amd): the current concepts of pathogenesis and prospects for treatment. Adv Clin Exp Med. 2015:24(6):1099-104.

23. Zarbin MA, Casaroli-Marano RP, Rosenfeld PJ. Age-related macular degeneration: clinical findings, histopathology and imaging techniques. Dev Ophthalmol. 2014;53:1-32.

24. Takayama $\mathrm{K}$, Kaneko $\mathrm{H}$, Terasaki $\mathrm{H}$, et al. Evaluation of short-term outcomes of intravitreal aflibercept injections for age-related macular degeneration using focal macular electroretinography. Retina. 2017;37(3):553-60.

25. Takayama K, Kaneko H, Kataoka K, et al. Short-term focal macular electroretinogram of eyes treated by aflibercept \& photodynamic therapy for polypoidal choroidal vasculopathy. Graefes Arch Clin Exp Ophthalmol. 2017;255(3):449-55.

26. Takayama K, Ito $\mathrm{Y}$, Terasaki $\mathrm{H}$, et al. Cross-sectional pupillographic evaluation of relative afferent pupillary defect in age-related macular degeneration. Medicine (Baltimore). 2016;95(39):e4978.

27. Rahman I. Oxidative stress and gene transcription in asthma and chronic obstructive pulmonary disease: antioxidant therapeutic targets. Curr Drug Targets Inflamm Allergy. 2002;1(3):291-315.

28. Babizhayev MA, Strokov IA, Lankin VZ, et al. The role of oxidative stress in diabetic neuropathy: generation of free radical species in the glycation reaction and gene polymorphisms encoding antioxidant enzymes to genetic susceptibility to diabetic neuropathy in population of type 1 diabetic patients. Cell Biochem Biophys. 2015;71(3):1425-43.

29. Beatty S, Koh H, Phil M, Henson D, Boulton M. The role of oxidative stress in the pathogenesis of age-related macular degeneration. Surv Ophthalmol. 2000:45(2):115-34.

30. Marazita MC, Dugour A, Marquioni-Ramella MD, Figueroa JM, Suburo AM. Oxidative stress-induced premature senescence dysregulates VEGF and CFH expression in retinal pigment epithelial cells: implications for age-related macular degeneration. Redox Biol. 2016;7:78-87.

31. Ye F, Kaneko H, Terasaki $H$, et al. Malondialdehyde induces autophagy dysfunction and vegf secretion in the retinal pigment epithelium in agerelated macular degeneration. Free Radic Biol Med. 2016;94:121-34.

32. Nojima J, Miyakawa M, Kodama M, et al. Measurement of the oxidation stress degree by the automated analyzer JCA-BM 1650. Jpn J Med Technol. 2010;59:199-207.

\section{Publisher's Note}

Springer Nature remains neutral with regard to jurisdictional claims in published maps and institutional affiliations.

\section{Ready to submit your research? Choose BMC and benefit from:}

- fast, convenient online submission

- thorough peer review by experienced researchers in your field

- rapid publication on acceptance

- support for research data, including large and complex data types

- gold Open Access which fosters wider collaboration and increased citations

- maximum visibility for your research: over $100 \mathrm{M}$ website views per year

At BMC, research is always in progress.

Learn more biomedcentral.com/submissions 